Revista de Matemática: Teoría y Aplicaciones 2(2): 17-33 (1995)

\title{
EL NUEVO ORDEN MUNDIAL Y EL CONSEJO DE SEGURIDAD DE LAS NACIONES UNIDAS
}

\author{
Raúl Alvarado Sibaja ${ }^{1}$
}

\begin{abstract}
Resumen
Las cuotas de poder de los miembros del Consejo de Seguridad de las Naciones Unidas son analizadas aquí desde una perspectiva matemática, utilizando la teoría de juegos. Se generalizan los resultados y se aplican a la situación actual. Se hacen algunas recomendaciones sobre la estrategia que deben adoptar los países pequeños, tales como las naciones centroamericanas.
\end{abstract}

\begin{abstract}
The quota power of UN Security Council members are analysed from a mathematical point of view, using Game Theory. Results are generalized and applied to the current situation. Some recommendations are stated about the strategy that small countries, like Central American countries, should follow.
\end{abstract}

\section{Introducción}

Pienso que no exagero al afirmar que los cambios ocurridos en la escena política mundial, durante estos primeros años de la década de los noventas, han sido los más importantes desde que terminó la Segunda Guerra Mundial. No me interesa analizar, ahora, las causas del derrumbe del bloque socialista, lo dejo a criterio de cada lector, simplemente lo asumo como un hecho. Súbitamente el mundo cambió y ahora se habla de un nuevo orden mundial. Pero, ¿cuál es ese nuevo orden? ¿Será acaso el que imaginaba el ahora expresidente Bush, donde los Estados Unidos mandaban y el resto del mundo obedecía?

Cada orden tiene sus propios mecanismos e instancias para expresarse. El "viejo orden", consecuencia de la segunda guerra mundial, se reflejaba entre otras instancias, en las Naciones Unidas; muy especialmente en el Consejo de Seguridad (CS) y en los mecanismos de toma de decisión de este Consejo; pues estos organismos son creados inmediatamente después de la Segunda Guerra Mundial. Más tarde, o más temprano, este nuevo orden, cualquiera que sea, buscará modificar las instancias y mecanismos actuales, para que éstos reflejen la nueva realidad política, económica y militar del mundo.

El propósito del presente trabajo es tratar de preveer cuáles serán esas modificaciones; y viendo la problemática desde la perspectiva de un pequeño país tercermundista como el

\footnotetext{
${ }^{1}$ Escuela de Ciencias de la Computación e Informática, Universidad de Costa Rica
} 
nuestro, señalar algunos aspectos importantes con respecto a los mecanismos de toma de decisión en el Consejo de Seguridad. Para lo cual haremos uso de los métodos de una rama de la matemática aplicada conocida como "teoría de juegos". Antes de entrar a lo antes mencionado, es necesario hacer un rápido recuento de los cambios; por esto en la sección 2 se dá un somero vistazo al orden emergente de la segunda guerra mundial. En la sección 3 se plantea el modelo matemático, basado en la teoría de juegos, que expresa dicha situación en el Consejo de Seguridad de las Naciones Unidas. Los principales cambios ocurridos en los cincuenta años posteriores a la guerra son señalados en la sección 4 . En la sección 5 se indica la reciente reactivación del CS. La sección 6 señala los nuevos actores y criterios que deben tomarse en cuenta para conformar un nuevo orden mundial. La generalización del modelo matemático para expresar esta nueva situación es presentada en la sección 7 . En la sección 8 se presentan los datos obtenidos con este modelo. La sección 9 contiene algunas conclusiones y la sección 10 indica algunas posibles extensiones del tema. Las tablas de datos son presentadas como un anexo, así como una breve explicación de la teoría de juegos matemáticos.

Es conveniente aclararle al lector que este no es un clásico artículo de matemática ni un clásico artículo de Ciencias Políticas, la realidad a veces no se deja encasillar tan fácilmente. Algunas de las afirmaciones que se hacen podrían ser consideradas como juicios de valor por algunos lectores ( lo cual a su vez es un juicio de valor); lo que sí puedo asegurar es que esas afirmaciones no están en evidente contradicción con los hechos históricos. En todo caso, el lector matemático, si así lo desea, puede omitir los párrafos de contenido político y quedarse sólo con el modelo matemático.

\section{El viejo orden}

Está claro que al finalizar la Segunda Guerra Mundial emergió un nuevo (en ese entonces) orden mundial, muy diferente del que existía antes de la guerra. La principal característica (pero no la única) de la nueva situación fue la aparición de dos bloques o polos de poder representados por el Pacto de Varsovia y la OTAN. La otra característica importante fue la desaparición (en ese momento) de Alemania y Japón como potencias mundiales. Como decía un general norteamericano, la OTAN fue creada para "to keep the Yankees in, the Russians out and the Germans down". (No hay cita formal para esta frase, la oí citar a un panelista en una mesa redonda en la televisión cultural norteamericana en 1990, a pesar de su aparente simplicidad, me parece que la frase tiene un tremendo poder de síntesis). La ONU y el Consejo de Seguridad son, en buena parte, reflejo del orden de cosas imperante en ese momento: La Asamblea General de la ONU tiene poco o ningún poder pues sus decisiones no son de acatamiento obligatorio (es decir, los no miembros del CS, el resto del mundo, está de espectador); prácticamente todo el poder lo tiene el Consejo de Seguridad; más concretamente, los 5 miembros permanentes (USA, Inglaterra, Francia, URSS-Rusia, China) que, en conjunto controlan más del $98 \%$ del poder como veremos más adelante. Se debe recordar que el criterio para designar a los 5 miembros permanentes fue el que eran los países vencedores (militarmente) de la Segunda Guerra Mundial. Por esas triquiñuelas de la guerra fría, durante muchos años China no fue miembro de la ONU y el puesto 
en el Consejo de Seguridad lo ocupó la diminuta Taiwan, pues los aliados reconocían al derrotado ejército de Chiang Kai-Chek, refugiado en Taiwan, como el legítimo gobierno de China.

Es conveniente recordar también en qué consiste el mecanismo mediante el cual el Consejo de Seguridad toma decisiones: Este Consejo está compuesto por 15 miembros, 5 permanentes y 10 no permanentes, los cuales son electos por la Asamblea General. Para que una moción sea aprobada, debe contar al menos con 9 votos a favor, pero entre los votos a favor deben estar todos los de los 5 miembros permanentes. En esto consiste el derecho a veto que tiene cualquiera de los 5 miembros permanentes; a uno de ellos le basta con votar NO para que la moción no se apruebe aunque tenga 14 votos a favor. Este derecho al veto es lo que convirtió al CS en un organismo prácticamente inoperante en el contexto de la guerra fría. En la sección 5 se ampliará sobre este tema. Lo anterior muestra claramente cómo las instancias ONU y Consejo de Seguridad y sus mecanismos de toma de decisión son un reflejo del orden imperante al finalizar la Segunda Guerra Mundial.

Antes de seguir adelante es conveniente resaltar algo que es obvio, pero que si se pasa por alto, hará más difícil entender los resultados y conclusiones al final del trabajo. Dos son las situaciones básicas que pueden presentarse en el CS. Primero, que al menos uno de los permanentes esté en contra de una moción, de aquí es donde derivan su inmenso poder. Segundo, que los cinco permanentes estén interesados en aprobar una moción, del hecho de que esos cinco votos no son suficientes es de donde derivan su misérrima cuota de poder los no permanentes.

\section{El viejo orden en números}

Un modelo matemático apropiado para analizar las relaciones de poder en el seno del Consejo de Seguridad, es modelar la situación como en juego cooperativo, y por consiguiente hacer uso de todas las herramientas de las que nos provee la teoría de juegos. Concretamente, de acuerdo con F. Roberts [12], podemos modelar el Consejo de Seguridad como el siguiente juego.( Se sugiere al lector no familiarizado con estos temas ver el anexo al final).

$$
v=(39 ; 7,7,7,7,7,1,1,1,1,1,1,1,1,1,1)
$$

Esto significa que en este juego participan 15 jugadores. Los 5 primeros tienen 7 votos cada uno, los 10 restantes sólo un voto cada uno. Una moción es ganadora sí y sólo sí, alcanza al menos 39 votos. Nótese que para que una moción sea aprobada necesita como mínimo, el voto de los 5 primeros miembros $(7 \times 5=35$ votos $)$, más 4 votos del segundo grupo.

Similarmente, cuatro miembros permanentes más los diez no permanentes sólo alcanzan $7 \cdot 4+10=38$ votos, los cuales no son suficientes para aprobar una moción.

Esto NO significa que los 5 primeros tienen 7 veces más poder que el resto. Es bien conocido en la teoría de juegos, que el poder real no está en proporción directa con el número de votos. Por ejemplo, considérese el juego $v=(51 ; 48,49,3)$, una rápida mirada revela que si 2 jugadores cualquiera se unen, obtienen la cuota de 51 o más votos. Esto significa que 
los 3 tienen igual poder, es decir, el 33,33\%, a pesar de que el primer jugador tiene 16 veces más votos, que el tercero, por ejemplo.

Un procedimiento bien probado para medir el poder en estas situaciones, es determinar, de todas las posibles coaliciones que se pueden formar, en cuántas de ellas un determinado jugador es pivote; es decir, que su salida convierta a esa coalición de ganadora en perdedora. No es del caso analizar aquí todos los detalles, una de las fórmulas más conocidas para medir el poder, es el llamado índice de Shapley [11]; el cual, para juegos simples se expresa como:

$$
\Phi_{i}[v]=\sum_{S \in \&_{i}} \gamma(s)(v(S)-v(S-\{i\})) \text { donde } \gamma(s)=\frac{(s-1) !(N-s) !}{N !}
$$

Donde $\&_{i}$ es el conjunto de coaliciones a las que pertenece el jugador $i$.

Pues como el juego es simple se tiene que $v(S)-v(S-\{i\})$ es igual a 1 si $i$ es pivote, e igual a 0 si no lo es.

$$
\Phi i[v]=\sum_{S \in \& i} \frac{(s-1) !(N-s) !}{N !}
$$

donde $N$ es el total de jugadores, $S$ es cualquier coalición para la cual el jugador $i$ es pivote, $s$ es el número de jugadores en $S$, y \& $i$ es ahora el conjunto de todas las coaliciones $S$ para las cuales $i$ es pivote.

Decimos que un jugador es pivote en una coalición si su retiro de la misma transforma esta coalición de ganadora en perdedora. Es decir $v(S)=1$ y $v(S-\{i\})=0$.

Aplicando el índice de Shapley al caso del Consejo de Seguridad se tiene que:

- $\Phi i[v]=0,001865$ para cualquier $i$ miembro no permanente

- $\Phi j[v]=0,1963$ para cualquier $j$ miembro permanente

NOTA: La suma del valor del índice de todos los jugadores siempre es igual a 1.

De esto se obtienen las siguientes conclusiones:

- El conjunto de los 10 miembros no permanentes tiene el 1,87\% del poder.

- El grupo de los 5 miembros permanentes tienen $(5 \cdot 0,1963=)$ el 98,13\% del poder.

- Un miembro permanente tiene $(0,1963 / 0,001865=) 105$ veces, más poder, que uno no permanente.

\section{4. ¿Qué ha cambiado?}

"Sobre el rencor de clase

floreció el amor ayer Lenin y Zza Zza Gabor

se casaban en New York"

Joaquín Sabina [13] 
Los dramáticos cambios ocurridos en estos últimos años en lo que fue el bloque socialista, no sólo han estremecido todos los andamiajes ideológicos, sino que son, sin duda, los cambios políticos más importantes de las últimas cuatro o cinco décadas; no debemos caer, sin embargo, en el error de creer que son los únicos. De 1945 a 1992 han sucedido varios hechos que han contribuído también a formar el nuevo orden; entre ellos:

a. La descolonización de una muchos territorios africanos, asiáticos, de Oceanía y el Caribe. Esto significó el emerger de gran cantidad de países, inexistentes antes de 1945.

b. Mención especial merecen ciertos casos como el de India, colonia británica en 1945 y, uno de los paises más grandes y poblados del mundo, en la actualidad.

c. Desde el punto de vista del escenario europeo, ya no es tan cierto que los alemanes están "down", ni los rusos "out", ni los yankees "in". Alemania de ser en 1945 un país destrozado y dividido es, en 1990 nuevamente, una potencia económica y unificada.

d. Algo similar podemos decir de Japón. El país que quedó asolado y ocupado en 1945 es, en 1990, una gran potencia económica.

e. Por supuesto, que el más espectacular es el caso de China; de ser un territorio despedazado y repartido entre las potencias coloniales europeas, ha pasado a ser, hoy día, un país unificado; el más poblado del mundo, que ya superó las hambrunas. Es potencia militar y está en los albores de ser una gran potencia económica, sino la primera. (Algunos analistas económicos consideran que para el 2025 ya lo será).

En conclusión, ya no podemos ver el mundo como un escenario en el que hay cinco jugadores y el resto son expectadores. El mundo de 1990 tiene nuevos jugadores y éstos no están presentes en las instancias de poder del viejo orden. Además de los organismos regionales como la OEA, la Comunidad Europea, la Unidad Africana, SEATO (South East Asia Treaty Organization), hay otros que han aparecido después de la creación de las Naciones Unidas y cuyo propósito, no es sino el corregir el desajuste entre las instancias de poder existentes y la realidad de estas décadas; me refiero, concretamente, a la organización de los No Alineados y al Grupo de los Siete. Los No Alineados trataron ser un intento de respuesta ante la debilidad de la Asamblea General de la ONU, y el G7 es una especie de CS de facto y sin los estorbos (China y Rusia) ni los elementos decorativos ( los 10 miembros no permanentes).

\section{Un gobierno mundial}

La idea de que haya un solo gobierno que ejerza su mandato sobre todos los habitantes de este planeta, no es nueva. Pero, era una idea manejada sólo por unos cuantos pensadores y, como algo que tal vez sucedería en un futuro muy lejano.

Aunque siempre ha habido países expansionistas que se atribuyen el derecho de mandar a otros países; no es sino hasta la aparición del Consejo de Seguridad en que de una manera explícita se asigna este rol a un organismo. 
Durante 45 años el CS ha sido un organismo casi inoperante. Como consecuencia de la guerra fría han sido muy pocas las mociones que han sobrevivido al tamiz del CS; o bien la URSS y China ejercían su derecho a veto, o bien lo hacían Estados Unidos, Inglaterra o Francia. Sin embargo, una consecuencia de la desaparición del bloque socialista y de la guerra fría parece ser la reactivación del CS.

Esta reactivación y este rol del CS es lo que lo hacen ahora muy importante para nosotros, lo cual justifica plenamente la necesidad de estudiarlo a profundidad.

La guerra de Irak, el caso de Somalia y la guerra entre las ex-repúblicas yugoeslavas presentan indicios de esa reactivación, además de su rol como órgano de gobierno mundial, al contrario de lo que acaecía en épocas anteriores, en estos casos se tomaron decisiones con relativa agilidad. Pero como señalábamos anteriormente, las condiciones del mundo actual no son las mismas que cuando se creó el CS.

De las secciones 4 y 5 concluímos que el recientemente reactivado Consejo de Seguridad no está en concordancia con la realidad política, económica y militar del mundo de las postrimerías del siglo XX. Para que este organismo asuma en verdad el rol de gobierno mundial, a la larga o a la corta tendrá que ser reestructurado. El resto de este trabajo se basa en esta presunción, y de lo que trata es de determinar cuál debe ser la estructura óptima (desde un punto de vista de un país como Costa Rica) que debe tener dicho consejo.

\section{Los nuevos jugadores}

\section{Criterios de selección}

Creo que es realista suponer que un nuevo Consejo de Seguridad mantendrá el hecho de que no todos sus miembros estarán en igualdad de condiciones, es decir, que se mantendrá el que haya miembros permanentes con algunos privilegios especiales como el derecho al veto. Asumimos que lo que variará es el ingreso de nuevos miembros permanentes y el aumento o disminución de los no permanentes. Lo que está por verse es cuántos miembros permanentes y cuántos no permanentes habrá. Creo que es también de sentido común asumir que para poder ser un miembro permanente se debe cumplir con algunos requisitos o criterios de selección. Algunos de estos criterios podrían ser:

- Cantidad de población

- Fortaleza de su economía

- Poder militar convencional

- Capacidad nuclear

- Región (geográfica, política y cultural) del mundo que representarían. Miembro permanente del CS en la actualidad.

Tomando en consideración lo anterior, una posible lista de regiones y una lista de posibles candidatos es la siguiente: 


\begin{tabular}{|c|c|}
\hline POSIBLES REGIONES & POSIBLES CANDIDATOS \\
\hline Europa Occidental & $\underline{\text { Francia }}, \underline{\text { Reino Unido, Alemania, Italia }}$ \\
\hline Europa Oriental & $\overline{\text { Rusia }}$ \\
\hline Mundo Arabe-Islam & Egipto, Arabia Saudita, Irán \\
\hline Africa Negra & Nigeria, Zaire, Sudáfrica \\
\hline Norteamérica (excluyendo México) & $\underline{\text { USA, Canadá }}$ \\
\hline América Latina & Brasil, México, Argentina \\
\hline Oceanía & Australia \\
\hline $\begin{array}{l}\text { China, Corea, Sureste Asiático } \\
\text { (cultura de influencia china) }\end{array}$ & $\underline{\text { China }}$ \\
\hline $\begin{array}{l}\text { India, Pakistán, Bangladesh } \\
\text { (cultura de influencia hindú) }\end{array}$ & India \\
\hline Resto de Asia (los países isleños) & Japón, Indonesia \\
\hline
\end{tabular}

La distribución anterior debe tomarse sólo como un ejemplo, no es el resultado de un estudio concienzudo sobre el tema.

\section{Generalización del modelo}

En la sección 3 vimos que el CS actual se puede modelar como el juego:

$$
v=(39 ; 7,7,7,7,7,1,1,1,1,1,1,1,1,1,1)
$$

Este tipo de juegos se conocen con el nombre de juegos cuota. en este caso el total de jugadores es $N=15$, la cuota es $q=39$ votos, hay $n=5$ miembros permanentes, con 7 votos cada uno, y los no permanentes tienen un voto cada uno.

Los valores 39 y 7 no son números mágicos sino que pueden ser calculados a partir de las reglas del juego (ver sección 2), a saber:

- una moción para ser aprobada necesita el apoyo de 9 miembros, y (regla 1)

- los 5 permanentes deben ser parte de los que apoyan la moción (regla 2).

Definamos nuestra notación para poder entender el caso particular y luego ver el modelo general.

$N$ es el total de jugadores

$n$ es el total de jugadores permanentes

$p$ es el número de votos que tiene cada uno de los jugadores permanentes

$t$ es el número de votos que tiene cada uno de los jugadores no permanentes

$q$ es la cuota de votos que debe tener una moción para ser aprobada

$S$ es una coalición de jugadores que apoya una determinada moción

$|S|=s$ es el número de jugadores que componen una coalición $S$

Entonces, para el caso particular tenemos que $N=15, n=5$ y $q=9$. De donde, los valores

de $q, t$ y $p$ deben ser tal que $5 p+(9-5) t \geq q$ (regla 1$)$, además, $(5-1) p+(15-5) t<q$ (regla 2), de donde tenemos que $4 p+10 t<5 p+4 t$. 
Sin pérdida de generalidad vamos a asumir que $t=1$, pues en realidad $p$ y $t$ representan la proporción en que deben estar los votos de un miembro permanente y uno no permanente. Por lo tanto $6<p$, de donde vemos que podemos darle a $p$ el valor de 7 . Sustituyendo los valores de $p$ y $t$ en las desigualdades anteriores tenemos que

$$
4 \cdot 7+10 \cdot 1<q \leq 5 \cdot 7+4 \cdot 1, \text { de donde } q=39 .
$$

Note que las desigualdades que sirvieron para calcular estos valores dependen fuertemente de la regla 1, pues la regla 2 no es otra cosa que el derecho a veto, para este caso se ve que 9 de 15 miembros representa el $60 \%$ de los miembros. Podemos entonces generalizar la situación anterior de la siguiente manera. Sea c el porcentaje del total de miembros que deben votar por una determinada moción para que sea aprobada; tenemos entonces que se deben cumplir las siguientes desigualdades:

$$
n p+(c N-n) t \geq q>(n-1) p+(N-n) t .
$$

En otras palabras, una coalición $S$ es ganadora si y sólo si $|S|=s \geq c N$ y todos los miembros permanentes pertenecen a $S$.

NOTA: El valor de $c$ debe ser tal que $c N>n$, pues si no fuera así eso significaría que los miembros permanentes por sí solos pueden alcanzar el porcentaje de miembros necesarios para que una coalición compuesta solo por ellos pueda aprobar una moción, lo cual implicaría que el poder de un miembro no permanente es cero, pues no sería pivote en coalición alguna. Vamos a asumir entonces esa condición com verdadera.

El juego $v$ puede ser entonces representado de la siguiente manera:

$$
v=(n p+(c N-n) t ; \underbrace{p, p, p, p, \ldots, p}_{n \text { veces }} \underbrace{t, t, t, t, t, \ldots, t)}_{N-n \text { veces }}
$$

Podemos entonces calcular el valor $\Phi i[v]$ para un miembro no permanente

$$
\Phi i[v]=\sum_{S \in \& i} \gamma(s)(v(S)-v(S-i)) \text { donde } \gamma(s)=\frac{(s-1) !(N-s) !}{N !}
$$

Hemos dicho anteriormente que un jugador es pivote para una coalición si su salida de la misma la convierte de ganadora en perdedora. Es decir, el número de miembros de la coalición debe ser muy cercano a la cantidad mínima de jugadores necesaria. Concretamente, para que i sea pivote en $\mathrm{S}$ es necesario que se den las siguientes condiciones:

- $S$ tenga "casi" $c N$ miembros, es decir ||$S|-c N| \leq 1$

- $i$ sea uno de ellos

- los $n$ miembros permanentes pertenezcan a $S$

- los restantes $c N-n-1$ miembros de $S$ sean escogidos entre los otros $N-n-1$ miembros no permanentes. 
¿Cuántas coaliciones $S$ satisfacen esas condiciones?. Las tres primeras condiciones son totalmente restrictivas, sólo la cuarta nos deja cierto grado de libertad, y no es sino la bien conocida pregunta: ¿De cuántas maneras puedo escoger $c N-n-1$ elementos de un conjunto de $N-n-1$ elementos?. La respuesta a esta pregunta no es otra que el número de combinaciones

$$
C(N-n-1, c N-n-1)=\frac{(N-n-1) !}{(c N-n-1) !(N-c N) !}
$$

Por lo tanto $\Phi i[v]$ se reduce a:

$$
\Phi i[v]=\frac{(N-n-1) !}{(c N-n-1) !(N-c N) !} \gamma(c N)
$$

Sustituyendo el valor de $(c N)$ y luego de una transformación algebraica, el valor de $\Phi i[v]$ se reduce a:

$$
\Phi i[v]=\frac{C(c N-1, n)}{N \cdot C(N-1, n)}
$$

El problema se reduce entonces a encontrar los valores de $c, n$ y $N$ tales que $\Phi i[v]$ sea máximo.

\section{Análisis de resultados}

Las tablas de datos presentadas al final, son el resultado de ejecutar un programa de cómputo que evalúa la fórmula $\Phi i[v]$ del párrafo anterior, para distintos valores de $N, n \mathrm{y}$ $c$. Esta función toma diferentes tripletas $(N, n, c)$ y con cada una de ellas calcula el valor $\Phi i[v]$. Hemos restringido el rango de valores de cada una de estas variables a lo que nos parece razonable, de acuerdo a la realidad política. Así el valor de $c$ lo restringimos a los valores $c=0,55,0,6,0,65$ y 0,75 ; pues no es razonable suponer que una moción pueda ser aprobada con menos de la mitad de los votos ( o sea $c<0,5$ ), ni que necesite más de las tres cuartas partes de los mismos ( es decir, $c=0,75$ ). El valor de $N$, el total de miembros del Consejo de Seguridad, lo restringimos a un rango entre 15 (número actual) y 30, pues un órgano de esta naturaleza con más de treinta miembros parece ser demasiado grande. Similarmente el valor de $n$, total de miembros permanentes, lo restringimos de 5 a 14, pues parece muy improbable que disminuya el número de miembros permanentes; al contrario, la tendencia es a que aumente este grupo, de la tabla de regiones y países de la sección 6 se desprende que los permanentes podrían aumentar hasta unos 12, de modo

que muy difícilmente sobrepasará los 14. De todos modos, variar estos rangos de valores es sumamente sencillo y el programa no tendrá dificultad alguna en calcular los datos. Creo que vale la pena desviarnos momentáneamente de la línea de desarrollo que llevamos y hacer un comentario sobre la escogencia de valores que hicimos en el párrafo anterior, pues ésta es una de esas sutiles diferencias entre la teoría de la matemática aplicada, y la construcción concreta de modelos matemáticos sobre algún aspecto del mundo real. Cuando se modela una situación real, el analista debe hacer uso al máximo de los aspectos 
particulares de esa situación, de modo que sin pérdida de precisión, se simplifiquen los cálculos matemáticos. Así en nuestro caso, la teoría de la matemática aplicada nos impulsaría a estudiar las fórmulas anteriores para valores de $c$ en el intervalo $[0,1]$; pero la realidad nos indica que podemos restringirlo al intervalo $[0,5,0,75]$; de la misma manera, en la realidad nunca se habla de reglas de aprobación que requieran, por ejemplo, del $62,37 \%$ de los votos. en otras palabras, no hay necesidad de estudiar todos los valores de c en el intervalo mencionado, por esto nos restringimos a los valores $c=0,55,0,6,0,65$ y 0,75. Típicamente las legislaturas piden que una moción se apruebe por mayoría simple (más de la mitad), o por mayoría calificada de al menos las dos terceras o las tres cuartas partes, es decir, $c=0,66$ y $c=0,75$. Fin de la disgreción.

Si nos atenemos únicamente a lo que arrojan los datos, y nos olvidamos momentáneamente de las realidades y las presiones políticas, es entonces clara cuál debe ser la estrategia de Costa Rica, partiendo del supuesto de que nuestro país nunca será un miembro permanente del Consejo de Seguridad, sino que a lo eventualmente puede aspirar es a ser un miembro temporal.

Esta mejor estrategia para CR tiene dos posibles componentes, dependiendo de si consideramos única y exclusivamente los intereses de nuestro país como nación individual, o si lo vemos como parte de un grupo, el grupo de los países que nunca serán miembros permanentes (ver el aparte de juegos con coaliciones a priori en el anexo sobre teoría de juegos).

En el primer caso CR, y cada uno de los países pequeños, debe procurar que el CS quede reestructurado del siguiente modo:

$N=16$, es decir, aumentar sólo en uno el número de miembros del CS

$n=5$, o sea, mantener el mismo número de miembros permanentes.

$c=0,75$, en otras palabras, que una moción necesite para ser aprobada, de las tres cuartas partes de los votos, y no de únicamente las dos terceras partes $(c=0,66)$ como sucede actualmente. Es conveniente aclarar que estos valores son los que optimizan globalmente ( en el conjunto de valores estudiados) a la función $\Phi i[v]$, y pueden ser encontrados luego de la última tabla de datos.

En este primer caso un miembro no permanente tendría $\Phi i[v]=9,615385$ o sea, poco más de 9 milésimos del poder, y el grupo de los miembros no permanentes tendría $(16-5)$. $9,615385=105,77$ es decir, el $10 \%$ del poder.

El segundo caso se presenta cuando consideramos a los miembros no permanentes como un gupo frente a los miembros permanentes. En este caso lo que más importa es el poder del grupo y no el poder individual de cada uno de sus miembros. En esta situación CR, y cada uno de los países pequeños, deben procurar que el CS quede reestructurado del siguiente modo:

$\mathrm{N}=30$, es decir, aumentar en 15 el número de miembros del CS

$n=5$, o sea, mantener el mismo número de miembros permanentes.

$c=0,75$, en otras palabras, que una moción necesite para ser aprobada, de las tres cuartas partes de los votos, y no de únicamente las dos terceras partes $(c=0,66)$ como sucede actualmente.

Como puede verse, este segundo caso difiere del primero en que el número de miembros debería ser de 30 . El poder individual de cada miembro no permanente sería de $\Phi i[v]=$ 
5,712 milésimas (menor que en el primer caso), pero el poder del grupo sería de $(30-5)$. $5,712=142,8$ milésimas, es decir, el grupo tendría casi el $15 \%$ del poder.

El lector puede fácilmente comparar estos datos con los presentados en la sección 3 .

\subsection{Cómo se usan las tablas presentadas en el anexo}

Sería muy iluso suponer que la reestructuración del CS se va a reducir a un simple juego matemático, en otras palabras, es casi imposible evitar que se amplíe el número de miembros permanentes y con derecho a veto, pues hay factores políticos y económicos de mucho peso empujando en esa dirección. Cuando esta reestructuración se presente, lo más probable es que se va a ir negociando y aprobando por etapas, mediante votación de la Asamblea General de la ONU. Es desde este punto de vista que tienen sentido los datos presentados en las tablas; pues con ellas se puede tener mejor idea sobre la bondad de cada una de las propuestas que vayan apareciendo en el proceso de negociación. Dicho de otra manera, si se mantienen las reglas generales del juego, cualquier propuesta que se presente puede ser reducida a una tripleta de valores $(N, n, c)$ que la representa; entonces la bondad de dicha propuesta puede ser leída en las tablas.

Por ejemplo, supongamos que se presenta la propuesta de reestructurar el CS para incluir a Japón, Alemania, Brasil, Nigeria y Australia como miembros permanentes, y mantener la regla de que una moción necesita los dos tercios del total de los miembros para ser aprobada. ¿Qué debe hacer CR en este caso? La propuesta se resume rápidamente así: el valor de $c$ es 0,66 por lo que puede leer la respuesta en la tabla $c=0,65$, el valor de $n$ sube de 5 a 10 , es decir $n=10$. Si leemos la columna $n=10$ en la tabla $c=0,65$ nos damos cuenta que el valor más alto de esa columna (0.082) se encuentra en la antepenúltima fila, es decir para el valor de $N=28$. Por lo tanto Costa Rica podría aceptar la propuesta con la condición de que el número total de miembros del CS se eleve a 28.

\section{Conclusiones}

Tal vez algunas personas todavía piensen que el tema de este artículo o es irrelevante, o estamos hablando de un futuro muy lejano, o no es más que un cuento de ciencia ficción. Sin embargo, además de lo señalado en la sección 4, hay indicios que indican lo contrario. El tema de este artículo en realidad lo vengo pensando desde 1991, luego de los dramáticos cambios en Europa del este y la guerra del Golfo Pérsico. De esa época al momento actual, en muchas ocasiones han aparecido comentaristas sobre la situación internacional, los cuales directa o indirectamente hacen referencia al tema del CS. Uno de los que más frecuentemente tocan el tema es Mijail Gorvachev, desafortunadamente es muy difícil tomar la cita bibliográfica de una noticia por televisión. Como ejemplo de lo anterior basta con indicar que hace unos pocos meses, el 13 de enero de 1994, en el Noticiero ECO de Teletica Canal 7 en horas de la mañana, presentaron una entrevista con una embajadora norteamericana (no fue posible tomar el nombre) ante un organismo internacional con sede en Europa, creo que ante la NATO; en esa entrevista esta señora manifiesta la posición, aparentemente con el respaldo de su gobierno, de que Alemania y Japón deben ser incorporados como miembros permanentes del CS. 
En el ámbito costarricense encontramos que algunos pensadores también se preocupan por este tema. El Grupo Soberanía manifiesta lo siguiente:

"NACIONES UNIDAS Y ORGANISMOS FINANCIEROS INTERNACIONALES

El control de los organismos internacionales y de los centros de decisión por grupos restringidos de naciones amenaza el orden internacinal y debe ser revisado.

Por un lado, los cinco miembros permanentes del Consejo de Seguridad no representan ya el mundo actual y el derecho a veto que ejercen se ha transformado en un elemento distorcionador de ese orden. Los países latinoamericanos reclaman un lugar como miembro permanente en el Consejo, al que deberían integrarse también otras naciones, de modo que ese organismo refleje de manera más equilibrada el nuevo orden internacional" [14]

Creo que con esto se demuestra la relevancia del tema. En cuanto al tratamiento matemático del mismo, aunque a algunos les pueda parecer ciencia ficción, la verdad es que es ciencia muy real. Se puede estar seguro de que, antes de hacer alguna propuesta a la comunidad internacional, los servicios de inteligencia de los países del primer mundo harán un estudio matemático sobre el tema, mucho más profundo que lo presentado en este trabajo.

Nuestro mundo es cada vez más interdependiente y competitivo, los diversos países se ven cada vez más obligados a competir entre sí y a la vez a cooperar entre sí. En otras palabras, pareciera que estamos entrando en un mundo en el cual hay competidores, pero no hay enemigos a destruir. Como se explica en el anexo sobre teoría de juegos, los modelos de juegos cooperativos son un instrumento excelente para analizar este tipo de situaciones de conflicto amistoso. El análisis que se ha hecho sobre el caso del Consejo de Seguridad, nos sirve como un ejemplo para mostrar como estas técnicas pueden ser un instrumento muy útil para el análisis de situaciones políticas y económicas, sin pretender sustituir los métodos usados corrientemente en las ciencias sociales.

\section{Extensiones}

El presente trabajo se basa en la presunción de que lo que va a suceder con el CS es una extensión directa de la situación actual, es decir, que hay dos tipos de miembros: los permanentes y que tienen el derecho a veto y el grupo de los no permanentes. Una idea muy interesante a explorar, es que puedan existir tres o cuatro categorías de miembros del CS. Esto es, las dos actuales más otra categoría de miembros, los cuales sean permanentes pero no tengan derecho a veto, o bien otra categoría, los cuales no sean permanentes pero sí tengan derecho a veto. Para estos casos el modelo matemático presentado anteriormente deja de ser funcional y hay que desarrollar uno nuevo, pero los conceptos básicos de la teoría de juegos siguen siendo válidos. 


\section{Anexo I. Breve presentación de la teoría de juegos.}

Con este anexo se pretende dar una breve explicación de los conceptos básicos de la teoría de juegos, de modo que los especialistas en ciencias sociales puedan formarse una idea sobre el tema, pero sin usar el lenguaje matemático.

La teoría de juegos se dio a conocer por primera vez en 1943, con la publicación de un famoso libro titulado "Theory of Games and Economic Behavior", escrito por uno de los grandes genios matemáticos de este siglo, el húngaro John Von Neumann, junto con el economista Oskar Morgenstern. De la contraportada de la tercera edición de este libro extraemos los siguientes comentarios:

"La posteridad posiblemente mirará este libro como uno de los mayores logros científicos de la primera mitad del siglo veinte". American Mathematical Society Bulletin.

"Uno no puede sino admirar la audacia de visión, la perseverancia en los detalles, y la profundidad de pensamiento mostrados en casi cada página del libro" American Economic Review.

"El principal logro de este libro reside, más que en sus resultados concretos, en haber introducido a la economía los instrumentos de la lógica moderna, y en usarlos con un impactante poder de generalización". Journal of Political Economy

"El lector terminará este libro con una riqueza de ideas para la aplicación y para la conversión de esta teoría en un instrumento fundamental de análisis para las ciencias sociales". American Journal of Sociology.

La fuerza de esos comentarios y las fuentes de los mismos son suficientes para presentar el libro. En el prefacio de la primera edición los autores dicen que "este libro contiene una exposición y varias aplicaciones de una teoría matemática de los juegos. La teoría ha sido desarrollada por uno de nosotros desde 1928 y ahora es publicada por primera vez en su totalidad. Las aplicaciones son de dos clases: Por un lado a los juegos propiamente dichos, y por otro lado a problemas económicos y sociológicos, los cuales, como esperamos mostrar, son mejor abordados desde esta dirección".

Casi se podría decir que la década de los cuarenta fue la década de von Neumann, pues no sólo definió la arquitectura de las nacientes computadoras (arquitectura que todavía hoy está vigente), sino que sus ideas de la teoría de juegos tuvieron una casi inmediata aplicación en los problemas de estrategia militar de la segunda guerra mundial. A partir de ese momento, los avances en el desarrollo de esta teoría han sido enormes, y sus aplicaciones se han generalizado a una gran variedad de campos. Casi que cualquier situación de toma de decisiones, para uno o múltiples atributos, en donde haya situaciones de conflicto, es suceptible de ser abordada con algún submodelo de esta teoría.

Es conveniente aclarar que los autores se refieren a juegos de salón y no a actividades atléticas. Las más inmediatas aplicaciones son al análisis de juegos tales como ajedrez, juegos de cartas tales como póker, bridge, blackjack, dados, diversos tipos de loterías, etc. En todos estos juegos participan dos o más jugadores, pero todos son suma-cero, es decir lo que alguien gana es porque otro lo pierde. Son especialmente conocidos los juegos suma-cero de dos personas, los cuales ya han sido completamente estudiados. En estos casos, como la lucha es frontal, no hay posibilidades de que se formen coaliciones entre los jugadores (pues solo hay 2) ni puede llegarse a acuerdos (pues no hay un botín a repartir). 
Ejemplos de ese tipo de situaciones pueden ser un juego de damas, o ajedrez, o "paris o nonis" o "piedra papel tijera", o dos candidatos enfrentados en una elección en la que sólo ellos dos participan, dos generales enfrentados en un campo de batalla, o un duelo entre dos aviones, dos empresas que compiten por un mismo mercado. Con algunas variantes, todos estos juegos se expresan matemáticamente como una matriz, y se aplican técnicas de programación lineal para encontrar las estrategias óptimas.

Existe una variante de este tipo de juegos, y es cuando levantamos el requisito de comportamiento racional a uno de los 2 jugadores, es decir este jugador juega sin interés de ganar. $\mathrm{Su}$ aplicación mas difundida es lo que se llama juegos contra la naturaleza. Ejemplos de estas situaciones se dan cuando alguien debe de tomar decisiones, cuyos resultados pueden verse afectados por factores climáticos de los cuales no se tiene certeza. Otro ejemplo ocurre cuando un médico debe decidir entre varios posibles tratamientos, sin tener certeza de cuál de varias posibles enfermedades es la que aqueja al paciente. Matemáticamente estos juegos se tratan muy similarmente a los juegos suma-cero dos personas, la variante está en que la naturaleza no tratará de usar la estrategia que nos cause más daño, es decir no tratará de maximizar su beneficio.

Saliéndonos del reino de los suma-cero, encontramos algunos juegos muy curiosos. Por ejemplo, supongamos que hay dos personas esperando un autobús y se acerca una tercera y les dice lo siguiente: "Si se logran poner de acuerdo en cómo repartírselos, les regalo estos cien colones" (o los partidos políticos $A$ y $B$ son archienemigos del partido $C$, si logran llegar a un acuerdo pueden derrotar al partido $C$ y tomar el directorio de la Asamblea). Es claro que estamos ante una situación dual de cooperación-conflicto, pues evidentemente a cada uno de los dos lo que más le agradaría es poder irse para su casa con los cien colones, y cada uno tratará de convencer al otro (conflicto) de que a él le debe corresponder una mayor parte, digamos 80 colones, pero por otro lado está el temor de salir sin nada si no llega a un acuerdo con su compañero-adversario, de ahí la necesidad de cooperar. En casos como éstos, en que desconocemos toda otra circunstancia, debemos suponer una simetría en la situación de los dos jugadores, por lo que rápidamente se llega al punto de equilibrio, la obvia repartición de 50 colones para cada uno.

El tipo de juego que nos interesa aquí, son las situaciones donde hay una determinada riqueza o bien a repartirse entre los jugadores, y hay tres o más jugadores involucrados. El hecho de que haya más de dos jugadores permite que algunos de ellos unan sus fuerzas para lograr inclinar el resultado del juego a su favor, es decir permite la formación de coaliciones. Una de las condiciones que debe tener una coalición es la de estabilidad, es decir, que tan susceptibles son los miembros de la misma, a ser tentados por mejores ofertas de otros jugadores que no están en la coalición. Es también necesario determinar cuál es el valor de cada una de las coaliciones, este valor se mide como la cantidad de la riqueza (es decir, la tajada del pastel) que la coalición puede garantizarse por sus propios medios. Si denotamos con $S$ a una coalición cualquiera $v(S)$ y denota entonces el valor de esa coalición. Un requisito que se pide para que el juego tenga sentido, es que el valor de la unión de dos coaliciones sea mayor o igual que la suma del valor de esas coaliciones por separado, matemáticamente esto se expresa como: $v(S \bigcup T) \geq v(S)+v(T)$. Este tipo de juegos reciben el nombre de juegos cooperativos y su tratamiento matemático es muy diferente de los juegos suma cero. 
Dadas esas condiciones generales, hay varios índices para medir qué tan poderoso es cualquiera de los jugadores en un juego cooperativo. Uno de los índices más usados es el índice o valor de Shapley, el cual se basa en la idea de contar para cuántas de todas las posibles coaliciones un jugador es realmente esencial, es decir que su salida convierte a esa coalición de ganadora en perdedora. Para no entrar en mucho detalle matemático, tal vez lo más conveniente es dar un pequeño ejemplo de cómo se calcula este índice en el caso de un juego parlamentario, donde hay tres jugadores $A, B$ y $C$ con 3,2 y 1 votos respectivamente, y en donde para aprobar una propuesta se requieren 5 votos. Supongamos que los jugadores van llegando a la coalición uno a la vez, y en diferentes posibles órdenes. ¿En cuántas de esas posibles situaciones la llegada de A convierte a la coalición de perdedora en ganadora?

\section{ORDEN}

$\begin{array}{lll}1 & A \mid B C & \text { Notación: Leyendo de izquierda a derecha } \\ 2 & A C \mid B & \text { la línea vertical indica el momento en que la } \\ 3 & B \mid A C & \text { coalición se convierte de perdedora en ga- } \\ 4 & B C \mid A & \text { nadora, al agregársele el jugador a la dere- } \\ 5 & C A \mid B & \text { cha de la línea. } \\ 6 & C B \mid A & \end{array}$

Puede observarse que la llegada de $\mathrm{A}$ es pivote en 3 casos $(3,4$ y 6$)$, la llegada de $B$ es pivote en 3 casos (1, 2 y 5); mientras que $C$ no lo es en ninguno. Por lo tanto el índice de Shapley para $A$ es de $3 / 6$, lo mismo para $B$ y para $C$ es de $0 / 6$. Es decir, A tiene el $50 \%$ del poder, lo mismo $B$, mientras que $C$ tiene cero poder.

Como sólo hay tres jugadores existen únicamente 6 casos por examinar, pero el número de casos crece velozmente con el número de jugadores. Por ejemplo, con 5 jugadores son 120 casos y con 6 jugadores son 720 casos. Todo esto requiere que se utilicen ciertas técnicas de conteo, pero esto no es necesario para entender las ideas esenciales. 


\section{Anexo II. Tablas de valores de la función $\Phi i[v]$}

Para el valor de $c=0,55$

Los valores verdaderos han sido multiplicados por mil NV significa resultado no válido

\begin{tabular}{cllllllllll}
\multirow{5}{*}{$N=15$} & $n=5$ & $n=6$ & $n=7$ & $n=8$ & $n=9$ & $n=10$ & $n=11$ & $n=12$ & $n=13$ & $n=14$ \\
& 0,699 & 0,155 & 0,019 & $\mathrm{NV}$ & $\mathrm{NV}$ & $\mathrm{NV}$ & $\mathrm{NV}$ & $\mathrm{NV}$ & $\mathrm{NV}$ & $\mathrm{NV}$ \\
& 0,437 & 0,087 & 0,010 & $\mathrm{NV}$ & $\mathrm{NV}$ & $\mathrm{NV}$ & $\mathrm{NV}$ & $\mathrm{NV}$ & $\mathrm{NV}$ & $\mathrm{NV}$ \\
& 0,754 & 0,206 & 0,041 & 0,005 & $\mathrm{NV}$ & $\mathrm{NV}$ & $\mathrm{NV}$ & $\mathrm{NV}$ & $\mathrm{NV}$ & $\mathrm{NV}$ \\
& 0,503 & 0,126 & 0,023 & 0,002 & $\mathrm{NV}$ & $\mathrm{NV}$ & $\mathrm{NV}$ & $\mathrm{NV}$ & $\mathrm{NV}$ & $\mathrm{NV}$ \\
& 0,774 & 0,238 & 0,060 & 0,011 & 0,001 & $\mathrm{NV}$ & $\mathrm{NV}$ & $\mathrm{NV}$ & $\mathrm{NV}$ & $\mathrm{NV}$ \\
& 1,084 & 0,387 & 0,119 & 0,030 & 0,005 & 0,001 & $\mathrm{NV}$ & $\mathrm{NV}$ & $\mathrm{NV}$ & $\mathrm{NV}$ \\
& 0,774 & 0,258 & 0,074 & 0,017 & 0,003 & 0,000 & $\mathrm{NV}$ & $\mathrm{NV}$ & $\mathrm{NV}$ & $\mathrm{NV}$ \\
& 1,032 & 0,387 & 0,129 & 0,037 & 0,009 & 0,001 & 0,000 & $\mathrm{NV}$ & $\mathrm{NV}$ & $\mathrm{NV}$ \\
& 0,763 & 0,269 & 0,084 & 0,022 & 0,005 & 0,001 & 0,000 & $\mathrm{NV}$ & $\mathrm{NV}$ & $\mathrm{NV}$ \\
& 0,981 & 0,381 & 0,135 & 0,042 & 0,011 & 0,002 & 0,000 & 0,000 & $\mathrm{NV}$ & $\mathrm{NV}$ \\
$\mathrm{N=30}$ & 0,745 & 0,275 & 0,092 & 0,027 & 0,007 & 0,001 & 0,000 & 0,000 & $\mathrm{NV}$ & $\mathrm{NV}$ \\
& 0,932 & 0,373 & 0,137 & 0,046 & 0,013 & 0,003 & 0,001 & 0,000 & 0,000 & $\mathrm{NV}$ \\
& 0,725 & 0,276 & 0,097 & 0,031 & 0,008 & 0,002 & 0,000 & 0,000 & 0,000 & $\mathrm{NV}$ \\
& 0,886 & 0,362 & 0,138 & 0,048 & 0,015 & 0,004 & 0,001 & 0,000 & 0,000 & 0,000 \\
& 0,702 & 0,275 & 0,100 & 0,033 & 0,010 & 0,003 & 0,001 & 0,000 & 0,000 & 0,000 \\
& 0,843 & 0,351 & 0,137 & 0,050 & 0,017 & 0,005 & 0,001 & 0,000 & 0,000 & 0,000
\end{tabular}

Los resultados parciales son: $\max =1,083591 \quad c=0,55 \quad n=5 \quad N=20$

Para el valor de $c=0,60$

Los valores verdaderos han sido multiplicados por mil NV significa resultado no válido

$\begin{array}{ccccccccccc} & \mathrm{n}=5 & \mathrm{n}=6 & \mathrm{n}=7 & \mathrm{n}=8 & \mathrm{n}=9 & \mathrm{n}=10 & \mathrm{n}=11 & \mathrm{n}=12 & \mathrm{n}=13 & \mathrm{n}=14 \\ \mathrm{~N}=15 & 1,865 & 0,622 & 0,155 & 0,022 & \mathrm{NV} & \mathrm{NV} & \mathrm{NV} & \mathrm{NV} & \mathrm{NV} & \mathrm{NV} \\ & 1,166 & 0,350 & 0,078 & 0,010 & \mathrm{NV} & \mathrm{NV} & \mathrm{NV} & \mathrm{NV} & \mathrm{NV} & \mathrm{NV} \\ & 1,697 & 0,617 & 0,185 & 0,041 & 0,005 & \mathrm{NV} & \mathrm{NV} & \mathrm{NV} & \mathrm{NV} & \mathrm{NV} \\ & 1,131 & 0,377 & 0,103 & 0,021 & 0,002 & \mathrm{NV} & \mathrm{NV} & \mathrm{NV} & \mathrm{NV} & \mathrm{NV} \\ & 1,548 & 0,595 & 0,198 & 0,054 & 0,011 & 0,001 & \mathrm{NV} & \mathrm{NV} & \mathrm{NV} & \mathrm{NV} \\ & 1,987 & 0,851 & 0,327 & 0,109 & 0,030 & 0,006 & 0,001 & \mathrm{NV} & \mathrm{NV} & \mathrm{NV} \\ & 1,419 & 0,568 & 0,203 & 0,062 & 0,016 & 0,003 & 0,000 & \mathrm{NV} & \mathrm{NV} & \mathrm{NV} \\ & 1,769 & 0,774 & 0,310 & 0,111 & 0,034 & 0,009 & 0,002 & 0,000 & \mathrm{NV} & \mathrm{NV} \\ & 1,308 & 0,538 & 0,202 & 0,067 & 0,019 & 0,004 & 0,001 & 0,000 & \mathrm{NV} \mathrm{NV} & \\ & 1,594 & 0,708 & 0,292 & 0,109 & 0,036 & 0,010 & 0,002 & 0,000 & 0,000 & \mathrm{NV} \\ & 1,884 & 0,892 & 0,397 & 0,163 & 0,061 & 0,020 & 0,006 & 0,001 & 0,000 & 0,000 \\ & 1,449 & 0,652 & 0,275 & 0,107 & 0,038 & 0,012 & 0,003 & 0,001 & 0,000 & 0,000 \\ & 1,691 & 0,805 & 0,362 & 0,153 & 0,059 & 0,021 & 0,007 & 0,002 & 0,000 & 0,000 \\ & 1,329 & 0,604 & 0,259 & 0,104 & 0,038 & 0,013 & 0,004 & 0,001 & 0,000 & 0,000 \\ \mathrm{~N}=30 & 1,533 & 0,733 & 0,333 & 0,143 & 0,057 & 0,021 & 0,007 & 0,002 & 0,001 & 0,000 \\ & 1,737 & 0,868 & 0,415 & 0,189 & 0,081 & 0,032 & 0,012 & 0,004 & 0,001 & 0,000\end{array}$


Los resultados parciales son: $\max =1,986584 \quad c=0,60 \quad n=5 \quad N=20$

Para el valor de $c=0,65$

Los valores verdaderos han sido multiplicados por mil NV significa resultado no válido

$\begin{array}{cllllllllll} & \mathrm{n}=5 & \mathrm{n}=6 & \mathrm{n}=7 & \mathrm{n}=8 & \mathrm{n}=9 & \mathrm{n}=10 & \mathrm{n}=11 & \mathrm{n}=12 & \mathrm{n}=13 & \mathrm{n}=14 \\ \mathrm{~N}=15 & & & & & & & & & & \\ & 1,865 & 0,622 & 0,155 & 0,022 & \mathrm{NV} & \mathrm{NV} & \mathrm{NV} & \mathrm{NV} & \mathrm{NV} & \mathrm{NV} \\ & 2,622 & 1,049 & 0,350 & 0,087 & 0,012 & \mathrm{NV} & \mathrm{NV} & \mathrm{NV} & \mathrm{NV} & \mathrm{NV} \\ & 3,394 & 1,543 & 0,617 & 0,206 & 0,051 & 0,007 & \mathrm{NV} & \mathrm{NV} & \mathrm{NV} & \mathrm{NV} \\ & 2,262 & 0,943 & 0,343 & 0,103 & 0,023 & 0,003 & \mathrm{NV} & \mathrm{NV} & \mathrm{NV} & \mathrm{NV} \\ & 2,838 & 1,310 & 0,546 & 0,198 & 0,060 & 0,013 & 0,002 & \mathrm{NV} & \mathrm{NV} & \mathrm{NV} \\ & 3,406 & 1,703 & 0,786 & 0,327 & 0,119 & 0,036 & 0,008 & 0,001 & \mathrm{NV} & \mathrm{NV} \\ & 2,433 & 1,135 & 0,487 & 0,187 & 0,062 & 0,017 & 0,003 & 0,000 & \mathrm{NV} \mathrm{NV} & \\ & 2,875 & 1,437 & 0,671 & 0,287 & 0,111 & 0,037 & 0,010 & 0,002 & 0,000 & \mathrm{NV} \\ & 2,125 & 1,000 & 0,437 & 0,175 & 0,062 & 0,019 & 0,005 & 0,001 & 0,000 & \mathrm{NV} \\ & 2,479 & 1,240 & 0,583 & 0,255 & 0,102 & 0,036 & 0,011 & 0,003 & 0,001 & 0,000 \\ & 2,826 & 1,487 & 0,744 & 0,350 & 0,153 & 0,061 & 0,022 & 0,007 & 0,002 & 0,000 \\ & 2,174 & 1,087 & 0,515 & 0,229 & 0,094 & 0,035 & 0,012 & 0,003 & 0,001 & 0,000 \\ & 2,459 & 1,288 & 0,644 & 0,305 & 0,136 & 0,056 & 0,021 & 0,007 & 0,002 & 0,000 \\ & 2,738 & 1,493 & 0,782 & 0,391 & 0,185 & 0,082 & 0,034 & 0,013 & 0,004 & 0,001 \\ & 2,171 & 1,133 & 0,566 & 0,270 & 0,121 & 0,051 & 0,020 & 0,007 & 0,002 & 0,001 \\ \mathrm{~N}=30 & 2,405 & 1,303 & 0,680 & 0,340 & 0,162 & 0,073 & 0,031 & 0,012 & 0,004 & 0,001\end{array}$

Los resultados parciales son: $\max =3,405573 \quad c=0,650000 \quad n=5 \quad N=20$

Para el valor de $c=0,75$

Los valores verdaderos han sido multiplicados por mil NV significa resultado no válido

$\begin{array}{ccccccccccc} & \mathrm{n}=5 & \mathrm{n}=6 & \mathrm{n}=7 & \mathrm{n}=8 & \mathrm{n}=9 & \mathrm{n}=10 & \mathrm{n}=11 & \mathrm{n}=12 & \mathrm{n}=13 & \mathrm{n}=14 \\ \mathrm{~N}=15 & & & & & & & & & & \\ & 8,392 & 4,662 & 2,331 & 0,999 & 0,333 & 0,067 & \mathrm{NV} & \mathrm{NV} & \mathrm{NV} & \mathrm{NV} \\ & 9,615 & 5,769 & 3,205 & 1,603 & 0,687 & 0,229 & 0,046 & \mathrm{NV} & \mathrm{NV} & \mathrm{NV} \\ & 6,222 & 3,394 & 1,697 & 0,754 & 0,283 & 0,081 & 0,013 & \mathrm{NV} & \mathrm{NV} & \mathrm{NV} \\ & 7,111 & 4,148 & 2,262 & 1,131 & 0,503 & 0,189 & 0,054 & 0,009 & \mathrm{NV} & \mathrm{NV} \\ & 7,906 & 4,865 & 2,838 & 1,548 & 0,774 & 0,344 & 0,129 & 0,037 & 0,006 & \mathrm{NV} \\ & 8,609 & 5,534 & 3,406 & 1,987 & 1,084 & 0,542 & 0,241 & 0,090 & 0,026 & 0,004 \\ & 6,149 & 3,689 & 2,108 & 1,135 & 0,568 & 0,258 & 0,103 & 0,034 & 0,009 & 0,001 \\ & 6,708 & 4,192 & 2,515 & 1,437 & 0,774 & 0,387 & 0,176 & 0,070 & 0,023 & 0,006 \\ & 7,212 & 4,666 & 2,916 & 1,750 & 1,000 & 0,538 & 0,269 & 0,122 & 0,049 & 0,016 \\ & 7,662 & 5,108 & 3,305 & 2,066 & 1,240 & 0,708 & 0,381 & 0,191 & 0,087 & 0,035 \\ & 5,823 & 3,678 & 2,248 & 1,322 & 0,744 & 0,397 & 0,198 & 0,092 & 0,038 & 0,014 \\ & 6,202 & 4,032 & 2,546 & 1,556 & 0,915 & 0,515 & 0,275 & 0,137 & 0,063 & 0,026 \\ & 6,547 & 4,365 & 2,837 & 1,792 & 1,095 & 0,644 & 0,362 & 0,193 & 0,097 & 0,045 \\ & 6,859 & 4,676 & 3,118 & 2,026 & 1,280 & 0,782 & 0,460 & 0,259 & 0,138 & 0,069 \\ \mathrm{~N}=30 & 5,440 & 3,548 & 2,258 & 1,398 & 0,839 & 0,485 & 0,270 & 0,143 & 0,071 & 0,033 \\ & 5,712 & 3,808 & 2,483 & 1,580 & 0,978 & 0,587 & 0,340 & 0,189 & 0,100 & 0,050\end{array}$

Los resultados parciales son: $\max =9,615385 \quad c=0,75 \quad n=5 \quad N=16$. 
Los resultados finales (en el ámbito estudiado) son:

$$
\max =9,615385 \quad c=0,75 \quad n=5 \quad N=16
$$

\section{Referencias}

[1] Alvarado R. (1980) "A game with a priori unions", Revista Ciencia y Tecnología. Vol.4, N1,2 pp. 21-36.

[2] Alvarado, R. (1985) "El problema del valor multiatributo. Una aproximación desde la teoría de juegos", Revista Ciencia y Tecnología. Vol.9, N.1-2 pp. 7-10.

[3] Alvarado, R. (1987) "Un algoritmo para la toma de decisiones en el caso de múltiples criterios", Revista Ciencia y Tecnología. Vol.11, N.1 pp. 37-42.

[4] Alvarado, R. (1991) Dicision support systems models based on multiattribute utility functions for decision making. Tesis para optar al grado de Master of Science, Universidad de Carolina del Sur.

[5] Alvarado, R. (1992) "Multiattribute decision problems: an algorithm for long matrices", Revista Ingeniería. Vol. 2, N.2, pp. 11-25.

[6] Davis, M. D. (1979) Teoría de juegos. Alianza Universidad. Madrid.

[7] Groennings, S. at el (1970) The study of Coalition Behavior. Holt-Rinehart. New York.

[8] Harsanyi, J. C. (1977) Rational Behavior and Bargaining Equilibrium in Games and Social Situations. Cambridge University Press, London.

[9] Hwang, C.; Yoon, K. (1981) Multiattribute Decision Methods and Applications. Lecture Notes in Economics and Mathematical Systems. Springer-Verlag, Berlin.

[10] Jones, A.J. (1980) Game Theory: Mathematical Model of Conflict. John Wiley \& Sons, New York.

[11] Owen, G. (1982) Game Theory. Second Edition. Academic Press, New York.

[12] Roberts, F. (1976) Discrete Mathematical Models. Prentice Hall, New Jersey.

[13] Sabina, J. (1990) "El muro de Berlín"(canción), del álbum Mentiras Piadosas. Ariola Eurodisc SA, New York.

[14] Soberanía, Grupo. (1995) "América pide sensatez y cordura a sus presidentes". Documento de divulgación, San José, Costa Rica, enero de 1995.

[15] Neumann, J. Von; Morgenstern, O. (1976) Theory of Games and Economic Behavior. John Wiley \& Sons, New York. 